\title{
EL GRUPO EPIDANTHUS, SUBGRUPO SELAGINELLA DE EPIDENDRUM
}

\author{
Eric Hágsater \& Elizabeth Santiago Ayala
}

\author{
Herbario AMO, Montañas Calizas 490, México, D.F. 11000, México \\ herbamo@prodigy.net.mx,www.herbarioamo.org
}

\begin{abstract}
Resumen. La revisión de una gran cantidad de ejemplares de herbario determinados como Epidendrum selaginella Schltr. que mostraban diferencias foliares evidentes comparadas con el ejemplar tipo que sirvió para describir esta especie ampliamente distribuida en Costa Rica y Panamá así como el análisis de los segmentos florales de los mismos permitieron reconocen 2 nuevas especies muy cercanas a Epidendrum selaginella y por mucho tiempo confundidas con esa especie centroamericana: Epidendrum astroselaginella y Epidendrum stenoselaginella. Se presenta una clave ilustrada.
\end{abstract}

ABSTRACT. The revision of a large number of herbarium specimens determined as Epidendrum selaginella Schltr. and which had evident foliar differences compared to the type served to prepare a detailed description of this widely distributed species in Costa Rica and Panama, and an analysis of the floral segments led to the recognition of two new species closely allied to Epidendrum selaginella, and confused with that Central American species: Epidendrum astroselaginella, and Epidendrum stenoselaginella. An illustrated key is provided.

Key words: Epidendrum selaginella, astroselaginella, stenoselaginella, Epidanthus, Costa Rica

Epidendrum, es considerado uno de los géneros más grandes de orquídeas neotropicales. Está constituido por unas 1500 especies distribuidas desde el sur de los Estados Unidos hasta el norte de Argentina (Hágsater 1985). En el transcurso de los años, muchos autores han intentado dividirlo en subgéneros (Barringer 1991), los cuales han llegado a reconocerse, en varios casos, como géneros (Williams 1940), basándose en características como el número de polinios, la presencia de tallos engrosados y/o la posición en la que se produce la inflorescencia. Con cerca de 300 especies secuenciadas en su ADN, hemos llegado a la conclusión de que se trata de un género monofilético en donde se pueden reconocer diversos grupos y sub grupos con características vegetativas similares (Hágsater \& Soto 2005).

En 1940, Williams, tomando en cuenta básicamente en el número de polinios, propuso al grupo Epidanthus como un género: Epidanthus L.O. Williams y transfirió a este género 3 especies de Epidendrum: E. paranthicum Rchb.f., E. muscicolum Schltr. y E. goniorhachis Schltr. Garay (1977) y Barringer (1991) también evaluaron este grupo, el primero opinando que efectivamente debía tratarse como género separado, y el segundo estableciéndolo como un subgénero de Epidendrum, y agregando una especie nueva. Con el estudio de las numerosas especies que integran el género Epidendrum, se ha demostrado que el número de polinios no representa una característica exclusiva del grupo Epidanthus. En el género Epidendrum, existen especies que tienen 2, 4 y hasta 8 polinios (Hágsater \& Santiago 2010).

El grupo Epidanthus, es de pocas especies y se caracteriza por tener plantas pequeñas, escandentes con tallos muy delgados, hojas cortas y angostas, algo carnosas con una lígula diminuta opuesta a la lámina foliar, inflorescencia apical, usualmente secundiflora con flores pequeñas. Está ampliamente distribuido desde el sur de México hasta Ecuador, con particular diversidad en Centro América. Actualmente, consideramos que está formado por 11 especies divididas en dos subgrupos: el subgrupo Epidanthus (que incluye a las especies que tienen hojas semiteretes, 2 polinios y el labelo generalmente 3-lobado, (con excepción de Epidendrum

\footnotetext{
* Este artículo fue preparado en el marco de las celebraciones del 40 Aniversario del Jardín Botánico Lankester.
} 

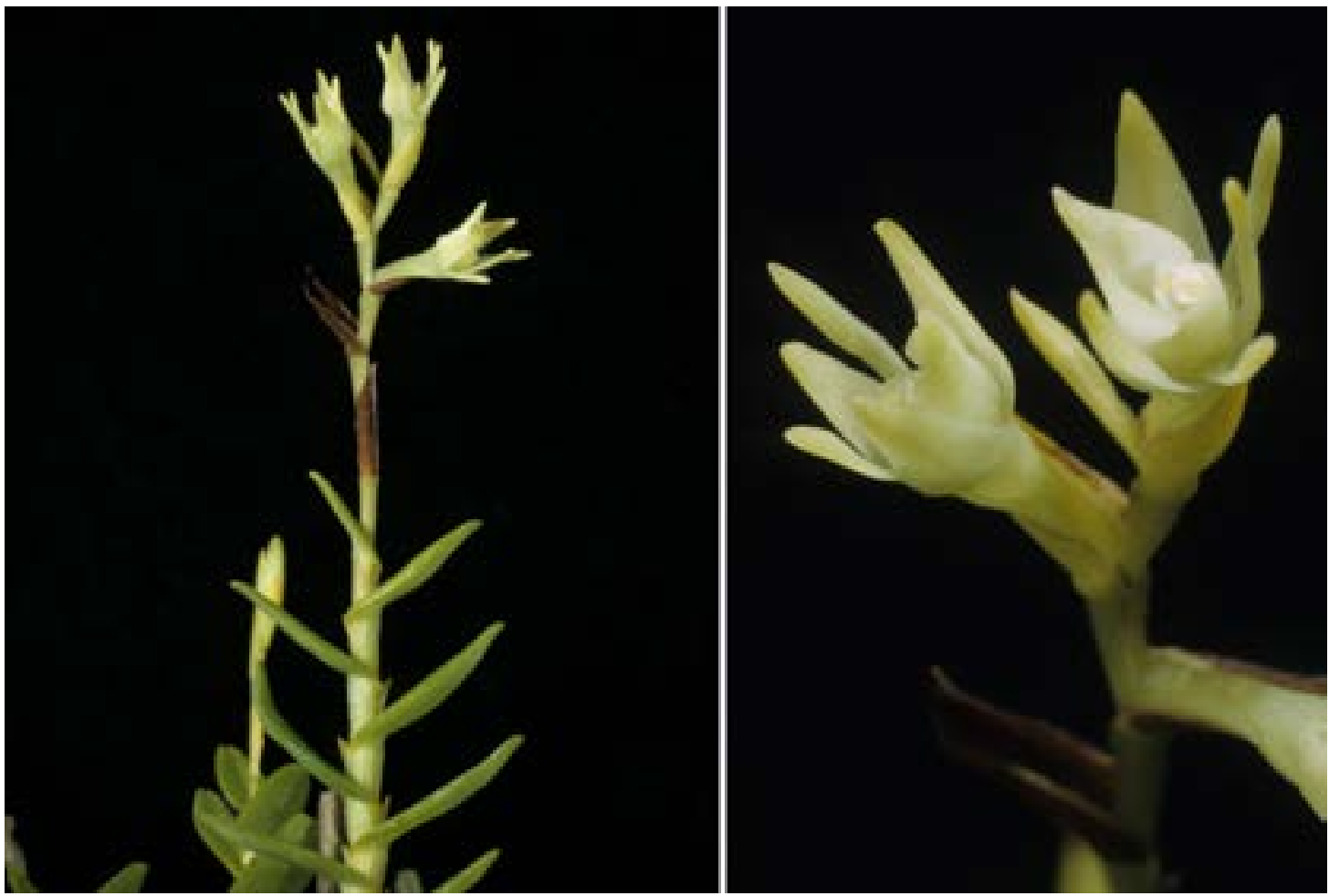

Figura 1. Epidendrum selaginella Schltr. basado en E.Hágsater 6537, Panamá: Cerro Horqueta, testigo: AMO A-589. Foto E. Hágsater.

goniorhachis que tiene el labelo entero), y el subgrupo Selaginella, que incluye a las especies con hojas planas, 4 polinios y de labelo entero, en el cual ésta incluido Epidendrum selaginella Schltr.

Revisando detenidamente una gran cantidad del material de herbario determinado bajo el nombre de Epidendrum selaginella (Fig. 1 y 4), encontramos 2 nuevas especies relacionadas, y por mucho tiempo confundidas con ésta especie bastante común y ampliamente distribuida en Costa Rica y Panamá.

Epidendrum selaginella fue descrita por Rudolf Schlechter en 1906 a partir de un ejemplar colectado por J. Cooper en Costa Rica: Se reconoce fácilmente por sus plantas pequeñas (de no más de $12 \mathrm{~cm}$ de alto, incluyendo la inflorescencia) con tallos tipo caña, hojas cortas y planas, generalmente ovadas con el ápice bilobado, con inflorescencias secundifloras de 5 a 7 pequeñas flores verdes o amarillo verdosas. Uno de los detalles que llamó nuestra atención cuando revisamos el material herborizado fue precisamente la forma de las hojas en algunos ejemplares determinados bajo el nombre de ésta especie, los cuales tenían hojas de forma distinta. La lámina foliar de estas plantas era claramente linear lanceolada y de una anchura menor a las hojas de E. selaginella (Santiago y Hágsater 2007; Fig. 4). Estudiando detenidamente éstos ejemplares, rehidratando flores e ilustrando los segmentos florales de varias de éstas plantas, encontramos además, que había diferencias también con respecto a las flores. En algunos ejemplares, las flores estaban laxamente distribuidas a lo largo del raquis y la mecánica de la antesis de los segmentos florales también era notoriamente distinta (la posición de los mismos era casi totalmente extendida). Notando que éstas características se mantenían de forma constante entre éstas plantas, en el año de 2007 procedimos a describir dos especies nuevas. Una bajo el nombre de Epidendrum astroselaginella Hágsater \& E.Santiago (Hágsater y Santiago 2007a; Fig. 2 y 5); la etimología del epíteto de ésta especie, hace referencia a la posición extendida de los segmentos florales y que recuerda a una estrella. Había otro pequeño grupo de ejemplares, de hojas angostas también, con la inflorescencia similar a Epidendrum selaginella, pero con diferencias 


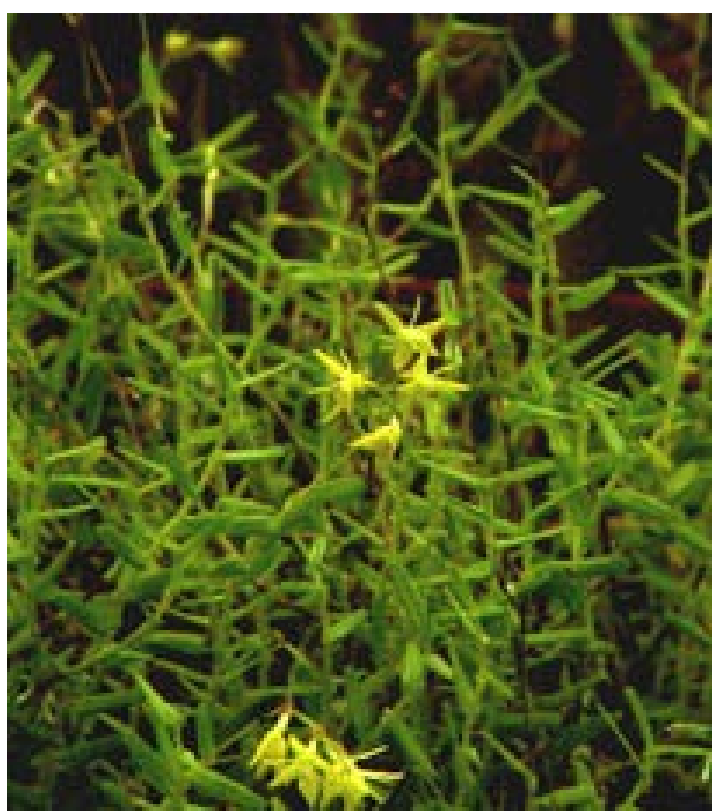

FIGURA 2. Epidendrum astroselaginella Hágsater \& E.Santiago basado en E.Hágsater s.n., Jardín Botánico Lankester, Costa Rica. Foto E. Hágsater. a nivel floral. A éste segundo grupo de colectas, de una distribución más amplia que va desde Nicaragua hasta Panamá (Fig. 7), lo describimos como Epidendrum stenoselaginella Hágsater \& E. Santiago (Hágsater y Santiago. 2007b; Fig. 3 y 6); el epíteto hace referencia a las hojas más angostas, comparada con E. selaginella.

\section{Clave de identificación DE LAS ESPECIES DEL GRUPO EPIDANTHUS, SUBGrupo SElaginElla}

El subgrupo Selaginella se caracteriza por sus hojas aplanadas, semejantes en tamaño y forma, generalmente cortas (2.7-10.5 mm de largo), el labelo entero y la antera con 4 polinios.

1 Hojas ovadas a oblongo ovadas, hasta $3.4 \mathrm{~mm}$ de ancho, flores distribuidas densamente hacia el ápice de la inflorescencia, ovario y dorso de los sépalos densamente papilosos; Costa Rica y Panamá

\section{E. selaginella Schltr.}

1' Hojas linear lanceoladas, hasta $1.9 \mathrm{~mm}$ de ancho, flores distribuidas laxamente en la inflorescencia, ovario y sépalos glabros
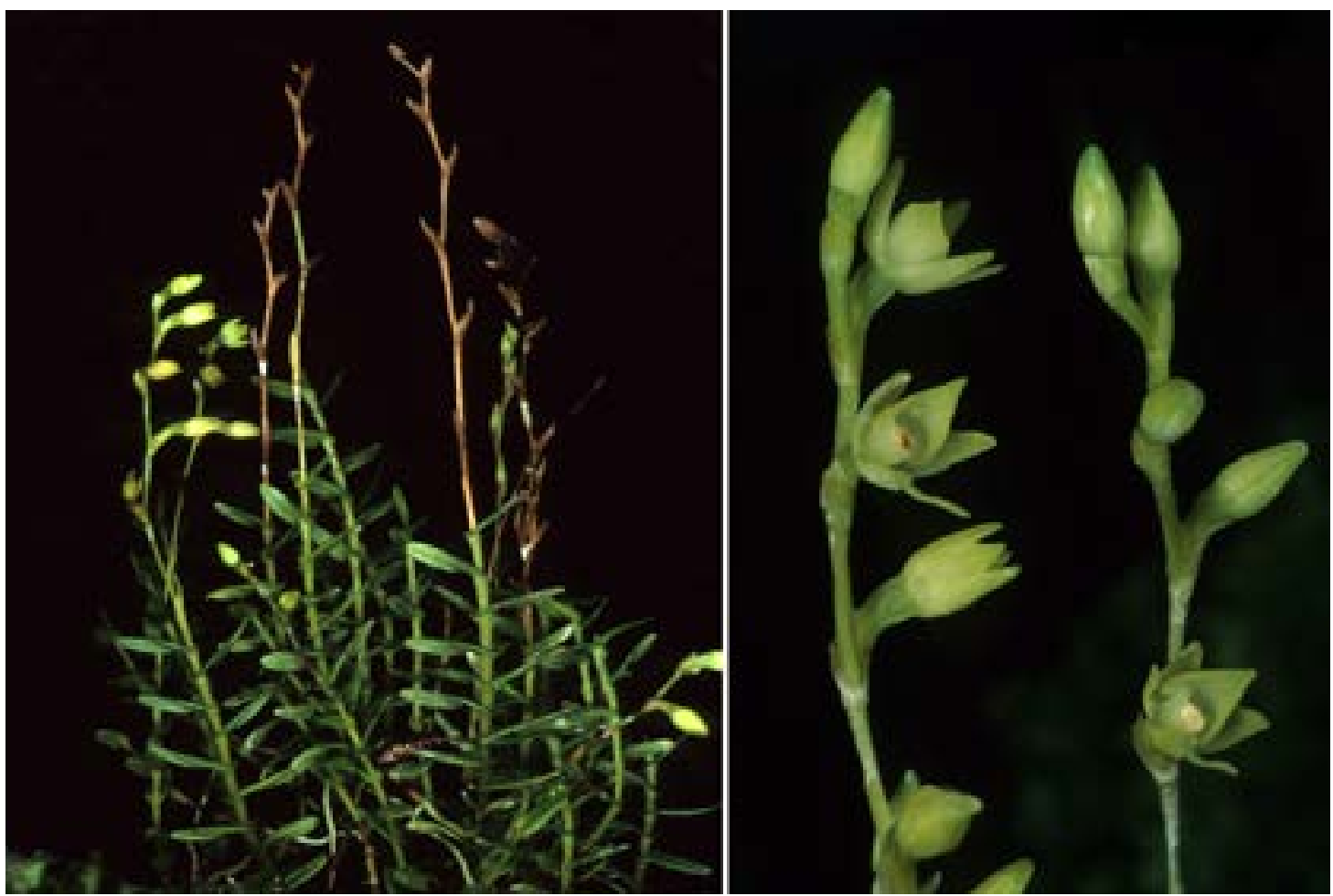

Figura 3. Epidendrum stenoselaginella Hágsater \& E.Santiago basado en E.Hágsater 11115; Costa Rica: Volcán Poás-San José, testigo: AMO 15886, holotipo. Foto: E. Hágsater. 

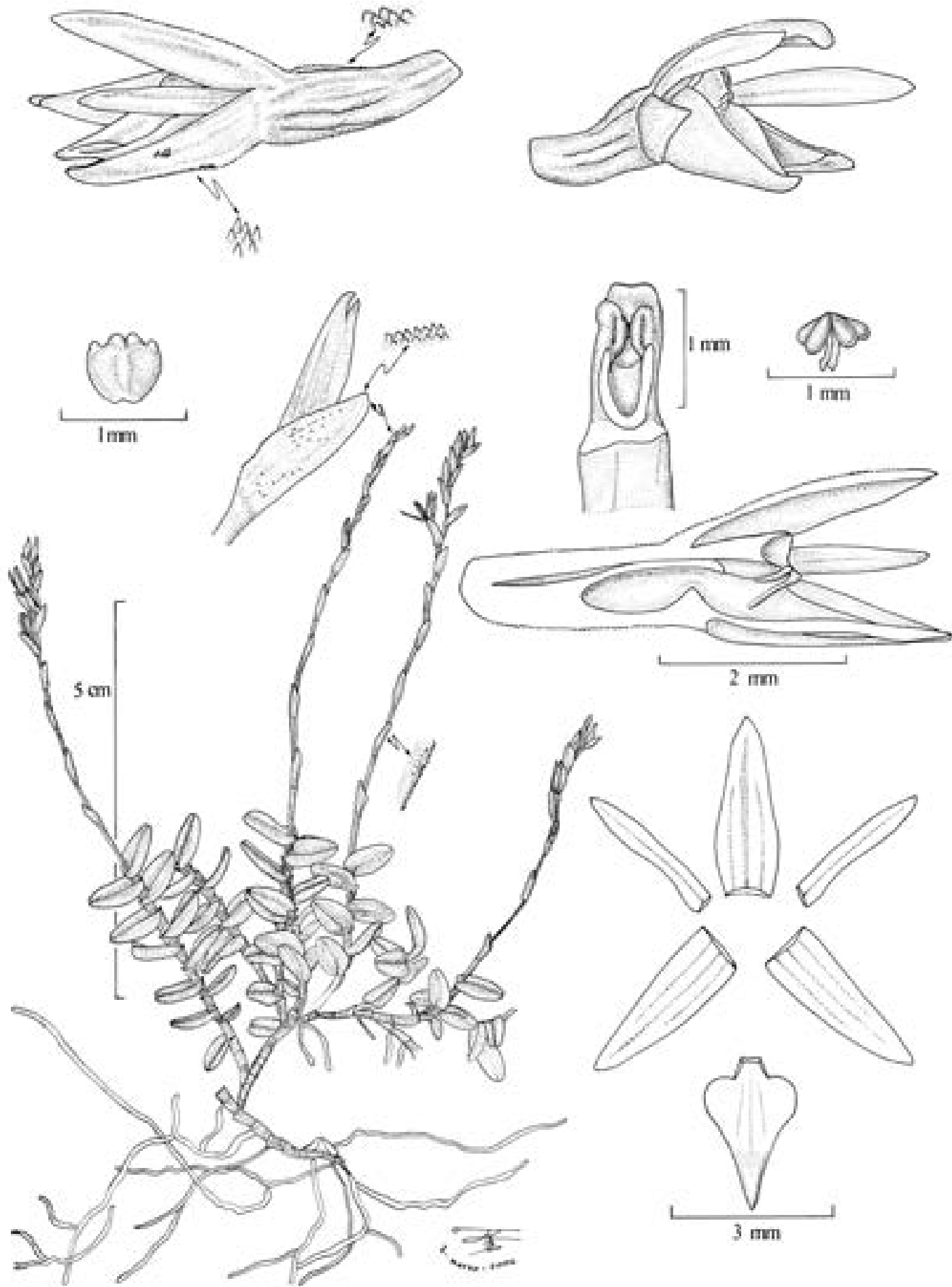

Figura 4. Epidendrum selaginella Schltr. basado en R.L.Dressler 5730; Panamá, Coclé, 8 km N of El Copé; testigo: AMO 2329. Dibujo de R. Jiménez Machorro. 


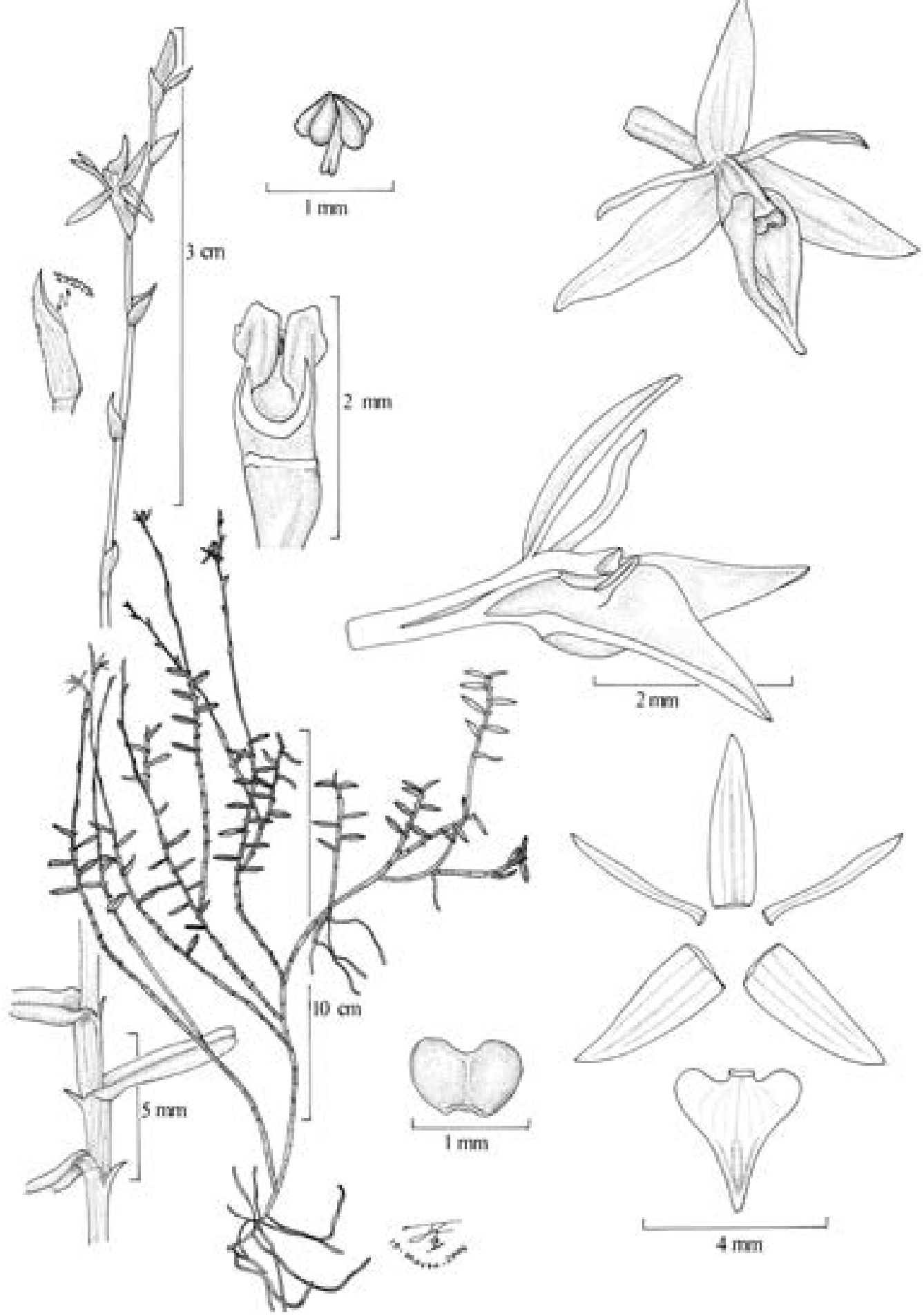

FigurA 5. Epidendrum astroselaginella Hágsater \& E.Santiago basado en L.D. Gómez P. 23814, Costa Rica, Cordillera de Talamanca; testigo: MO 3586978, holotipo. Dibujo de R. Jiménez Machorro. 


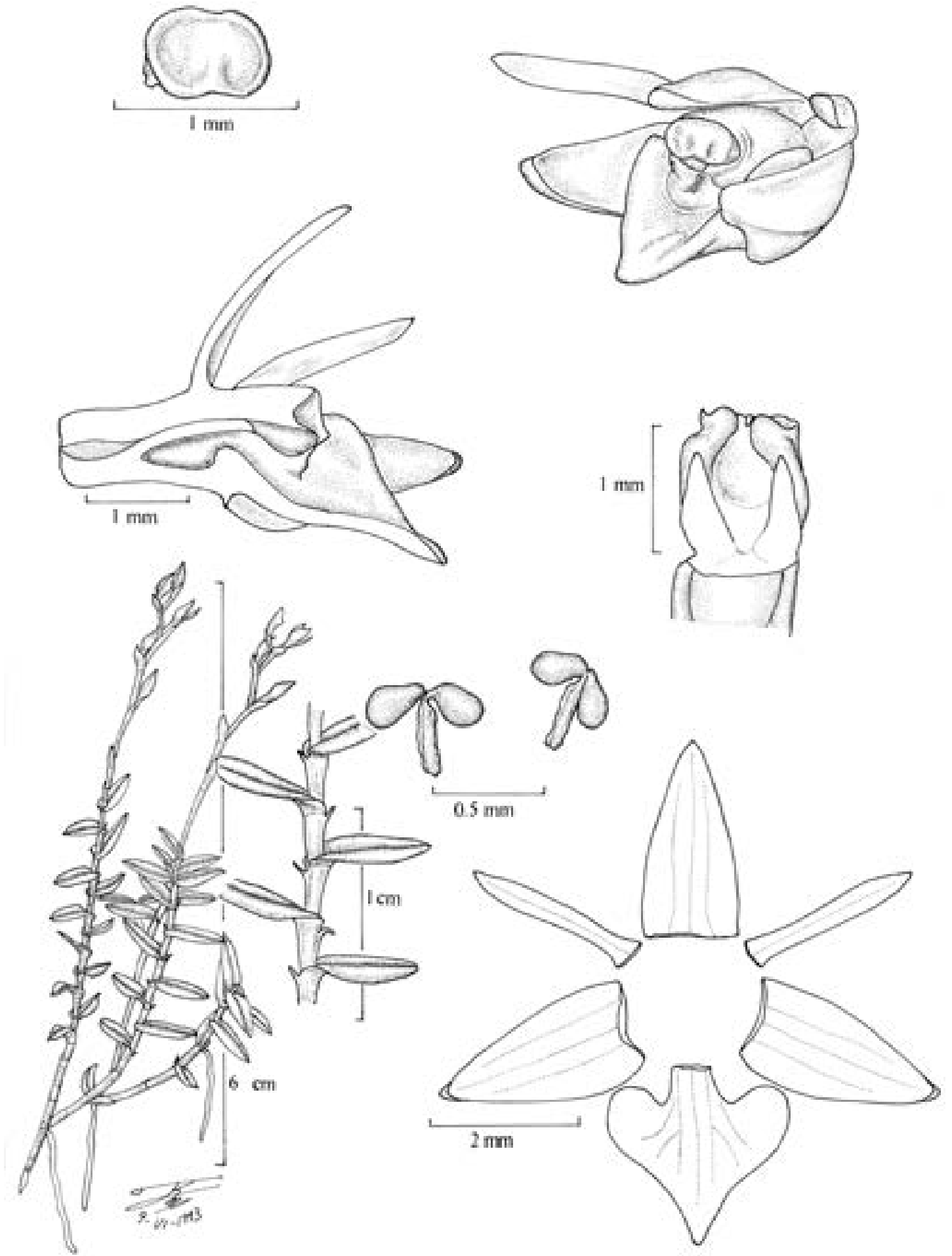

Figura 6. Epidendrum stenoselaginella Hágsater \& E.Santiago basado en E.Hágsater 11115, Costa Rica, Volcán Poás-San José; testigo: AMO 15886, holotipo. Dibujo de R. Jiménez Machorro. 


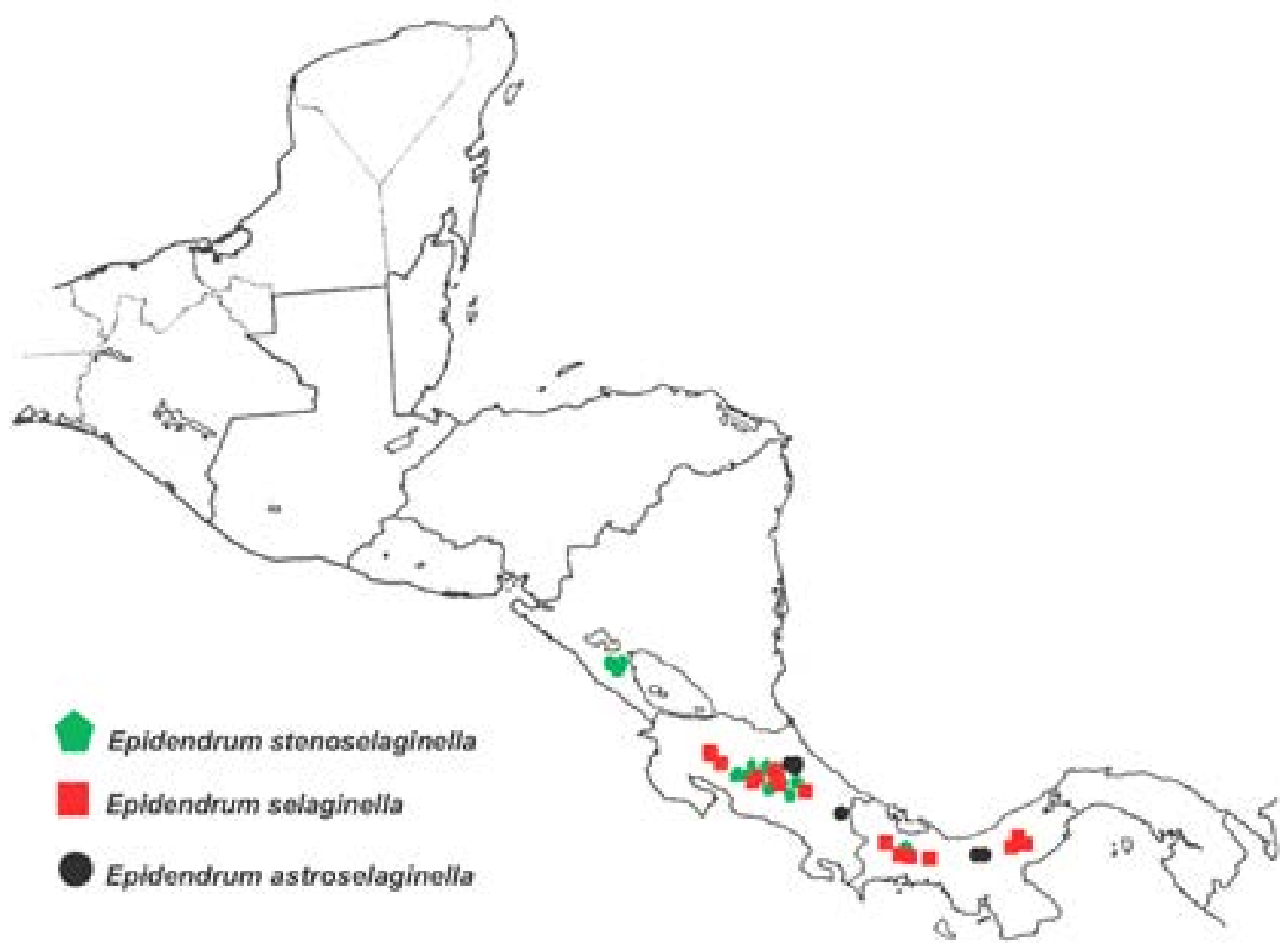

FIgURA 7. Mapa de distribución de Epidendrum selaginella, E. stenoselaginella y E. astroselaginella.

2 Segmentos florales extendidos, sépalos 3.5-4.2 $\mathrm{mm}$ de largo, labelo proporcionalmente más largo que ancho, columna con un par de dientes laterales prominentes; Costa Rica y Panamá

E. astroselaginella Hágsater \& E.Santiago

2' Segmentos florales entreabiertos, sépalos 2.5-3 mm de largo, labelo proporcionalmente tan largo como ancho, columna con un par de dientes laterales incipientes; Nicaragua, Costa Rica y Panamá

E. stenoselaginella Hágsater \& E.Santiago

\section{LiTERATURA CITADA}

Barringer, K. 1991. A revision of Epidendrum subgenus Epidanthus (Orchidaceae). Brittonia 43(4): 240-252.

Garay, L.A. 1977. The Subtribe Epidanthinae L. O. Wms. The Orchid Digest, 41(1): 19-22.

Hágsater, E. 1985. Towards an understanding of the genus Epidendrum. En K.W. Tan, (ed.), Proceedings of the $11^{\text {th }}$ World Orchid Congress: $195-199$. Miami, U.S.A.
Hágsater, E. y M.A. Soto. 2005. Epidendrum. En A.M. Pridgeon, P.J. Cribb, M.W. Chase y F.N. Rasmussen (eds.). Genera Orchidacearum 4: Epidendroideae, part one: 236-251. Oxford University Press, Cambridge, U.K.

Hágsater, E. y E. Santiago. 2007a. Epidendrum astroselaginella. En Hágsater, E. \& L. Sánchez Saldaña (eds.), The Genus Epidendrum, Part 6, Species New and Old in Epidendrum. Icon. Orchid. 9: t. 917.

Hágsater, E. y E. Santiago. 2007b. Epidendrum stenoselaginella. En Hágsater, E. \& L. Sánchez Saldaña (eds.), The Genus Epidendrum, Part 6, Species New and Old in Epidendrum. Icon. Orchid. 9: t. 991.

Hágsater, E. y E. Santiago. 2010. Epidendrum peristerium. En Hágsater, E. \& L. Sánchez Saldaña (eds.), The Genus Epidendrum, Part 9, Species New and Old in Epidendrum. Icon. Orchid. 13: t. 1370.

Santiago, E. y E. Hágsater. 2007. Epidendrum selaginella. En Hágsater, E. \& L. Sánchez Saldaña (eds.), The Genus Epidendrum, Part 6, Species New and Old in Epidendrum. Icon. Orchid. 9: t. 984.

Williams, L.O. 1940. A new genus of the Orchidaceae from Central America. Bot. Mus. Leafl. 8: 148-151. 
APÉNDICE. Clave ilustrada de identificación de las especies del grupo Epidanthus, subgrupo Selaginella.

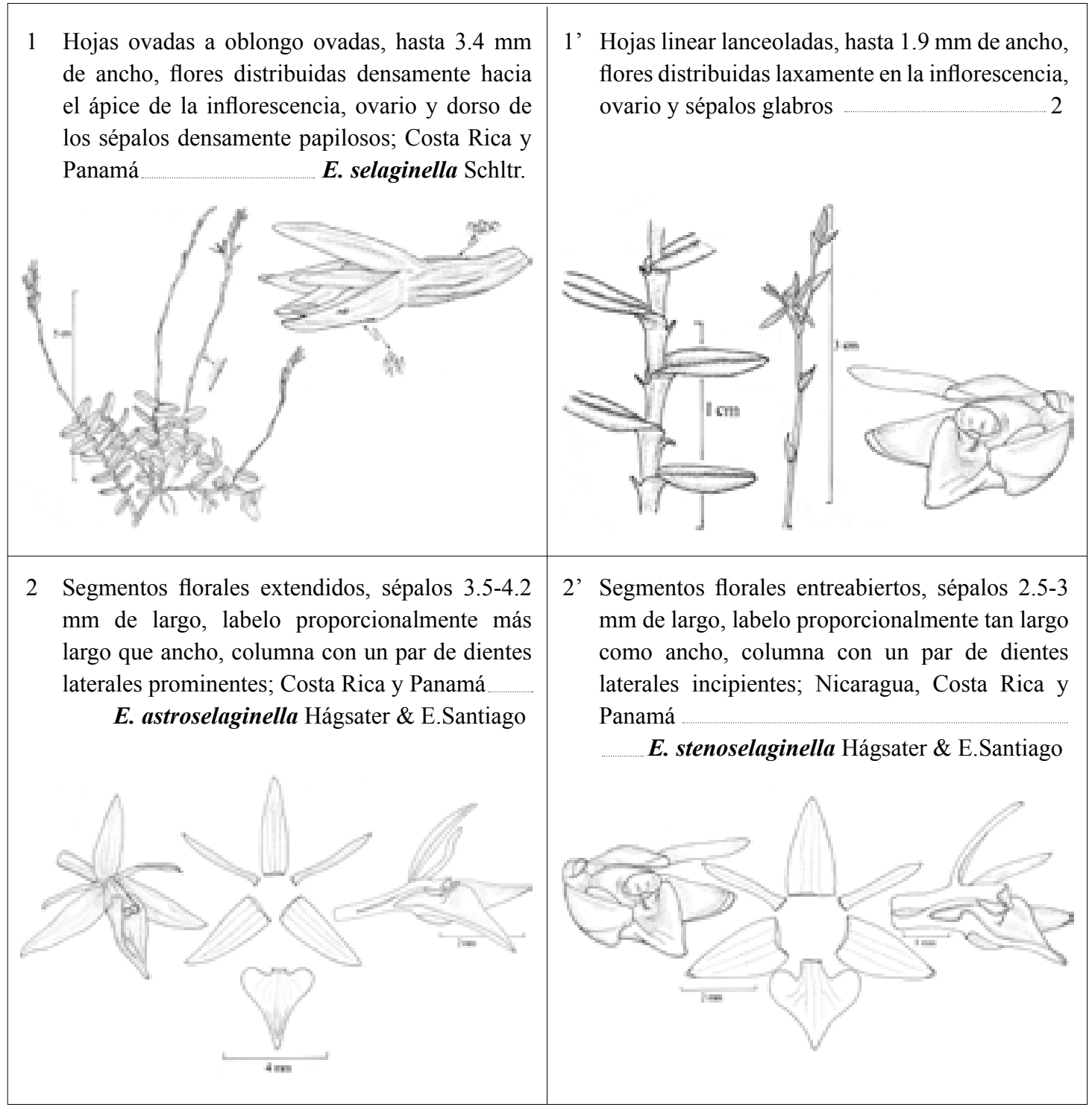

